\title{
Sausage oscillations of coronal loops
}

\author{
D. J. Pascoe ${ }^{1}$, V. M. Nakariakov ${ }^{1}$, and T. D. Arber ${ }^{1}$ \\ Physics Department, University of Warwick, Coventry, CV4 7AL, UK \\ e-mail: D. J.Pascoe@warwick.ac.uk
}

Received 6 July 2006 / Accepted 29 September 2006

\begin{abstract}
Aims. Analytical theory predicts the existence of trapped global (or fundamental) sausage fast magnetoacoustic modes in thick and dense coronal loops only, with the periods estimated as the ratio of double the loop length and the Alfvén speed outside the loop. We extend this study to the leaking regime, considering global sausage modes of long loops with small density contrasts.

Methods. Anti-symmetric fast magnetoacoustic perturbations (sausage, or $m=0$ modes) of a low $\beta$ plasma slab with the symmetric Epstein profile of plasma density are modelled numerically.

Results. It was found that long loops with sufficiently small density contrast can support global sausage leaky modes of detectable quality. The periods of the leaky modes are found to be approximately determined by the loop length and the external Alfvén speed. If the loop length can be estimated from imaging observations, the observed period of this mode provides us with the information about the Alfvén speed outside the loop. For typical flaring coronal loops, the estimated periods of the global sausage modes are about 5-60 s.
\end{abstract}

Key words. Sun: corona - Sun: oscillations - Sun: flares - magnetohydrodynamics (MHD)

\section{Introduction}

Magnetohydrodynamic (MHD) waves in the solar corona attract attention in the context of the coronal heating and solar wind acceleration problems (see, e.g. Aschwanden 2004; Ofman 2005, for recent reviews) and as a tool for remote diagnostic of the coronal plasma (Nakariakov \& Verwichte 2005, for recent review). One of the MHD modes of a coronal loop is a so called sausage mode (see Fig. 1), also known in the coronal literature as an $m=0$ mode, radial mode, varicose mode and fast magnetoacoustic mode. This mode is an anti-symmetric perturbation to the transverse velocity profile of the loop, causing the variation of the loop minor radius and hence the subsequent contractions and widenings of the loop tube. This mode does not perturb the axis of the tube. The sausage mode is essentially compressible, but, in contrast with the acoustic (or longitudinal) mode, the mode perturbs the plasma mainly in the transverse direction.

Observationally, the sausage mode can be seen in EUV and soft X-ray bands as periodic variation of the thermal emission intensity caused by the density perturbations and as the periodic Doppler broadening of emission lines, caused by the LOS velocities in the transverse motions of the plasma. Also, in flaring loops filled by non-thermal electrons, this mode can modulate microwave emission by the variation of the absolute value of the magnetic field in the emitting volume. In addition, in flaring loops of variable cross-section, this mode can modulate hard $\mathrm{X}$-ray and white light emission from the loop footpoints, by periodic change of the mirror ratio at the loop legs, causing the periodic increase and decrease in the precipitating non-thermal electrons (Zaitsev \& Stepanov 1982).

First identification of a spatially resolved sausage mode was made by Nakariakov et al. (2003) with the Nobeyama Radioheliograph. They found an in-phase variation of the $17 \mathrm{GHz}$ and $34 \mathrm{GHz}$ emission along a thick and short flaring loop, which had a well pronounced spectral peak near $17 \mathrm{~s}$. The signal was strongest at the loop top. It was concluded that the global (or fundamental) sausage mode was observed. A more detailed study of this event confirmed that conclusion (Melnikov et al. 2005).

The standard approach in the theoretical description of sausage modes is based upon modelling of the oscillating loop as a magnetic cylinder or a magnetic slab, confined between two rigid walls corresponding to the surface of the Sun (see Fig. 2). MHD modes of a magnetic cylinder were first determined by Zaitsev \& Stepanov (1975) and Roberts et al. (1984), and of a magnetic slab by Edwin \& Roberts (1982). In particular, there can be modes which are guided by those structures, which are evanescent outside the cylinder or the slab. Such modes are called trapped modes. Also, there can be leaky modes, which radiate the energy in the external medium in the direction transverse to the cylinder or the slab. For low $\beta$ plasmas, typical for the corona, it was found that there are trapped magnetoacoustic modes in magnetic cylinders and slabs if the external Alfvén speed exceeds the internal Alfvén speed. According to the model, there can be two sausage modes, fast and slow, in coronal loops. However, as the slow sausage mode does not produce any noticeable perturbation of the loop minor radius, we classify that mode as a longitudinal mode (Nakariakov \& Verwichte 2005) and apply the term sausage to the fast mode only. In the cylinder approximation, the phase speed of the trapped sausage mode is situated between the Alfvén speed values inside and outside the loop, $C_{\mathrm{A} 0}$ and $C_{\mathrm{Ae}}$ respectively, and decreases with increasing wave number. Figure 3 shows a typical dependence of the phase speed of the sausage mode upon the normalised wave number. For smaller wave numbers, the phase speed increases up to the value of the external Alfvén speed, where it experiences a cut-off. For wave numbers smaller than the cut-off value sausage modes become leaky. Sausage modes 


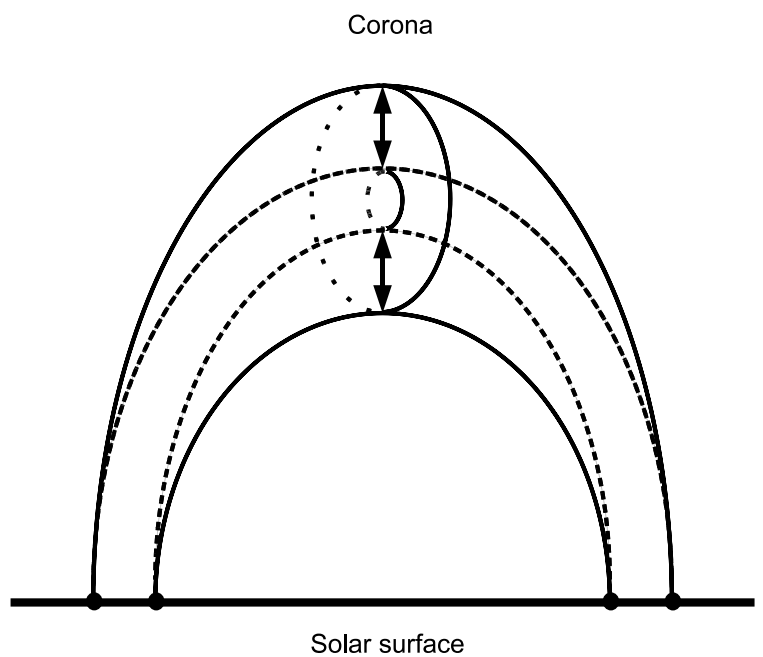

Fig. 1. The sausage mode of a coronal loop is an anti-symmetric perturbation to the transverse velocity profile of the loop, causing the variation of the loop minor radius and hence the subsequent contractions (dashed curves) and widenings (solid curves) of the loop tube. This mode does not perturb the axis of the tube.

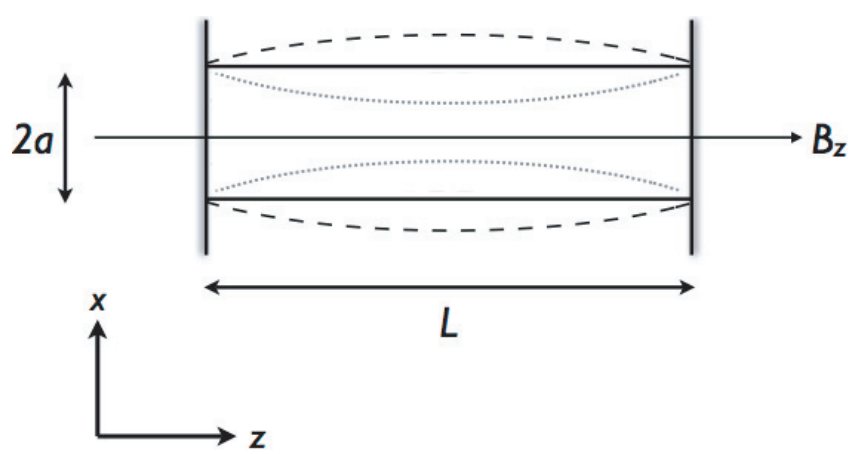

Fig. 2. A loop is modelled as a straight slab of density enhancement in a low $\beta$ plasma with a constant equilibrium magnetic field. The sausage mode is a compressive mode which perturbs the plasma mainly in the transverse $(x)$ direction. The global sausage mode has a wave length of twice the loop length $L$. The solid horizontal lines show the slab boundaries in the equilibrium case. The dashed and dotted curves show the maximum and minimum perturbation of the slab cross-section by the global sausage mode, respectively.

of a magnetic slab, which is a Cartesian counterpart of the cylinder model, demonstrate similar behaviour. Moreover, sausage modes of a slab with a smooth profile of the Alfvén speed, modelled by a symmetric Epstein profile (see Fig. 4), were found to have similar properties (Nakariakov \& Verwichte 2005).

First theoretical studies of sausage modes of coronal structures (Rosenberg 1970; Zaitsev \& Stepanov 1975; Roberts et al. 1984) estimated the period of the global mode as a function of the loop minor radius. However, Nakariakov et al. (2003) (see also Aschwanden et al. 2004) established that, at least in the trapped regime, the period of the global sausage mode $P_{\mathrm{GSM}}$ is determined by the loop length $L$,

$P_{\mathrm{GSM}}=\frac{2 L}{C_{\mathrm{ph}}}$,

where $C_{\mathrm{ph}}$ is the specific phase speed of the sausage mode corresponding to the wave number $k=\pi / L, C_{\mathrm{A} 0}<C_{\mathrm{ph}}<C_{\mathrm{Ae}}$. The length of the loop $L$ should be smaller than $\pi / k_{\mathrm{c}}$ where $k_{\mathrm{c}}$ is the cut-off wave number. As Eq. (1) describes only trapped modes,

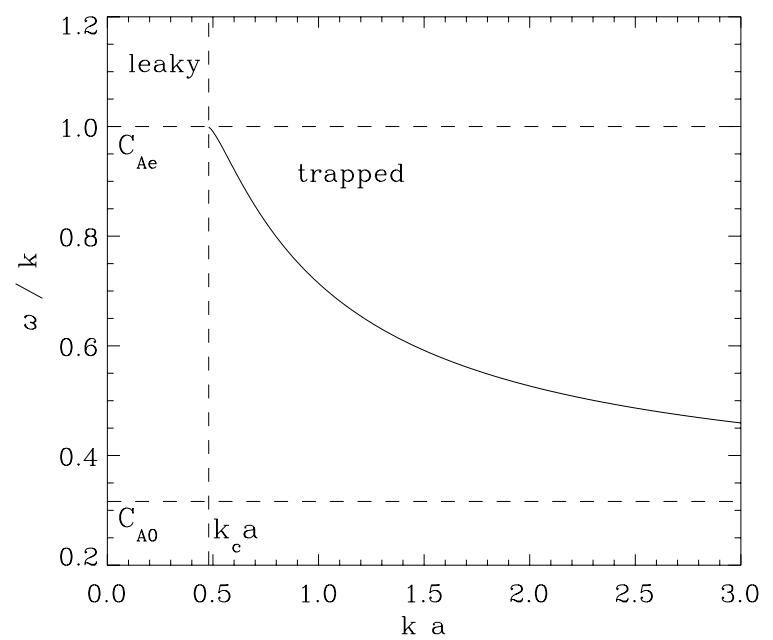

Fig. 3. A typical dispersion relation for a trapped sausage mode of a cylindric magnetic structure, showing the dependence of the phase speed $\omega / k$, normalised by the external Alfvén speed, upon the longitudinal wave number $k$, normalised by the structure's half-width $a$. The sausage mode dispersion curve has a cut-off when the phase speed equals the external Alfvén speed, which defines the regions of trapped and leaky solutions.

the above theory was shown to be applicable to global sausage oscillations in thick dense loops (Aschwanden et al. 2004).

It is still unclear whether in the small wave length limit $(k \rightarrow$ 0 ) the period of the global leaky sausage mode is determined by the loop minor radius, as suggested by Kopylova et al. (2002), or the formalism developed by Nakariakov et al. (2003) for the trapped mode can be extended to the leaky regime and hence be applicable to thin faint loops as well.

The aim of this paper is to establish the dependence of the period of global sausage modes upon the loop parameters by direct full-MHD numerical modelling. In Sect. 2 we describe the model used in the numerical simulations. A parametric study of the dependence of the global sausage mode period and rate of mode leakage on loop length and density contrast ratio are presented in Sect. 3 .

\section{Sausage modes of a magnetic slab}

In this work, a coronal loop is modelled as a straight slab of enhanced density, which is in pressure balance with the surrounding plasma. The equilibrium magnetic field is straight in the $z$ direction and constant. The equilibrium density profile is given by the symmetric Epstein function (Nakariakov \& Roberts 1995),

$\rho(x)=\left(\rho_{0}-\rho_{\mathrm{e}}\right) \operatorname{sech}^{2}(x / a)+\rho_{\mathrm{e}}$

where $\rho_{0}$ is the density at the loop axis, $\rho_{\mathrm{e}}$ is the external density far from the loop, $\rho_{\mathrm{e}}<\rho_{0}$ and $a$ is a parameter governing the inhomogeneity width such that the width of the loop is approximately $2 a$. The transverse density profile is shown in Fig. 4. As a result of the profile, the Alfvén speed increases from $C_{\mathrm{A} 0}$ at the centre to $C_{\mathrm{Ae}}$ at infinity.

In the zero $\beta$ case, the fast magnetoacoustic eigenfunctions (Cooper et al. 2003) which describe the perturbation of the transverse $(x)$ component of the plasma velocity in the sausage mode are given by the expression

$U(x)=\frac{\sinh (x / a)}{\cosh ^{\lambda}(x / a)}$, 


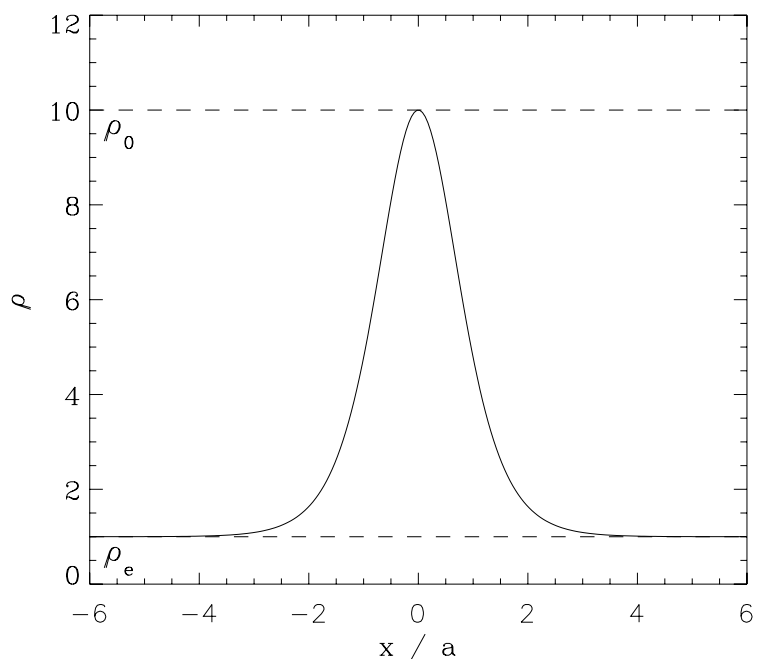

Fig. 4. A possible transverse dependence of the equilibrium plasma density, given by a symmetric Epstein profile. For this profile it is possible to derive the dispersion relation analytically.

where $\lambda=\left(C_{\mathrm{Ae}}^{2}-C_{\mathrm{p}}^{2}\right)^{1 / 2}|k| a / C_{\mathrm{Ae}}+1, k$ is the longitudinal wave number, and $C_{\mathrm{p}}=\omega / k$ is the phase speed, determined from the dispersion relation

$\frac{|k| a}{C_{\mathrm{A} 0}^{2}}\left(C_{\mathrm{p}}^{2}-C_{\mathrm{A} 0}^{2}\right)-\frac{2}{|k| a}=\frac{3}{C_{\mathrm{Ae}}} \sqrt{C_{\mathrm{Ae}}^{2}-C_{\mathrm{p}}^{2}}$.

Nakariakov et al. (2003) derived the necessary condition for the existence of the trapped global sausage mode of a straight cylinder,

$L / 2 a<\pi C_{\mathrm{Ae}} / 2 j_{0} C_{\mathrm{A} 0}$,

where $L$ is the loop length and $j_{0} \approx 2.40$ is the first zero of the Bessel function of the zeroth order, $J_{0}$. This condition requires a sufficiently thick and dense loop to support the mode. For a slab with the symmetric Epstein profile of density, used in this study, the cut-off wavenumber is given by

$k_{\mathrm{c}}=\frac{1}{a} \sqrt{\frac{2 C_{\mathrm{A} 0}^{2}}{C_{\mathrm{Ae}}^{2}-C_{\mathrm{A} 0}^{2}}}$.

Thus, the longest possible period of the trapped sausage mode is

$P_{\mathrm{GSM}}^{\max }=\frac{2 \pi}{C_{\mathrm{Ae}} k_{\mathrm{c}}}=\frac{\sqrt{2} \pi a \sqrt{C_{\mathrm{Ae}}^{2}-C_{\mathrm{A} 0}^{2}}}{C_{\mathrm{Ae}} C_{\mathrm{A} 0}}$

In the following, the wave length corresponding to the cut-off wave number $k_{\mathrm{c}}$ will be referred to as $L_{\mathrm{c}}$, where $L_{\mathrm{c}}=\pi / k_{\mathrm{c}}$. Hence, in the case of the Epstein profile, the periods of trapped standing sausage modes can only be shorter than $P_{\mathrm{GSM}}^{\max }$. On the other hand, the period of the global sausage mode is determined by the length of the loop by Eq. (1). Near the cut-off $k_{\mathrm{c}}$, the period of the trapped global sausage mode is given by the expression $P_{\mathrm{GSM}}=2 L / C_{\mathrm{Ae}}$. Consequently, in a slab with the symmetric Epstein profile of density, the trapped global sausage mode can exist only in sufficiently short and dense loops only:

$\frac{L}{a}<\frac{\pi \sqrt{C_{\mathrm{Ae}}^{2}-C_{\mathrm{A} 0}^{2}}}{\sqrt{2} C_{\mathrm{A} 0}} \approx \frac{\pi}{\sqrt{2}} \sqrt{\frac{\rho_{0}}{\rho_{\mathrm{e}}}}$,

similar to the cylindrical case with the step function profile of density (Aschwanden et al. 2004).

\section{Numerical modelling}

We performed numerical simulations of global sausage mode oscillations in terms of the slab model shown in Fig. 2 with the use of Lare2d (Arber et al. 2001). The governing equations simulated by the code are the (normalised) ideal MHD equations with an adiabatic equation of state. The effects of gravity are ignored.

$\frac{\mathrm{D} \rho}{\mathrm{D} t}=-\rho \nabla \cdot \boldsymbol{v}$

$\rho \frac{\mathrm{D} \boldsymbol{v}}{\mathrm{D} t}=(\nabla \times \boldsymbol{B}) \times \boldsymbol{B}-\nabla P$

$\frac{\mathrm{D} \boldsymbol{B}}{\mathrm{D} t}=(\boldsymbol{B} \cdot \nabla) \boldsymbol{v}-\boldsymbol{B}(\nabla \cdot \boldsymbol{v})$

$\rho \frac{\mathrm{D} \epsilon}{\mathrm{D} t}=-P \nabla \cdot \boldsymbol{v}$

where $\boldsymbol{v}$ is the velocity, $\boldsymbol{B}$ is the magnetic field, $P$ is the thermal pressure, $\epsilon=P / \rho(\gamma-1)$ is the internal energy density $(\gamma=5 / 3$ is the specific heat ratio) and $\rho$ is the mass density.

Lare $2 \mathrm{~d}$ operates by taking a Lagrangian predictor-corrector time step and after each Lagrangian step all variables are conservatively re-mapped back onto the original Eulerian grid using Van Leer gradient limiters. The code was designed for the simulation of nonlinear dynamics of low $\beta$ plasmas with steep gradients.

The equilibrium magnetic field was taken to be straight. The boundary conditions were line-tying in the background magnetic field direction $z$, at $z=-L / 2, L / 2$, corresponding to the boundary conditions at the coronal base. In the transverse direction, open boundary conditions were applied. The equilibrium density was taken to be uniform in the $z$ direction and had a symmetric Epstein profile (Fig. 4) in the transverse direction. In the transverse direction, the total pressure is kept constant by introducing an appropriate temperature profile. The plasma $\beta$ is fixed at 0.001 internally and externally.

A global sausage mode perturbation was applied to the transverse plasma velocity with the form

$v_{x}=A U(x) \cos (k z)$

where $k=\pi / L$ for the global standing mode. The transverse structure of the perturbation, $U(x)$, was given by Eq. (3), with the phase speed of $\omega / k=C_{\mathrm{Ae}}$ in all runs. The initial amplitude $A=$ $10^{-3}$ was always sufficiently small to avoid nonlinear effects.

In all simulations the loop semi-width was $a=3 \mathrm{Mm}$. The density contrast ratio varied, $\rho_{0} / \rho_{\mathrm{e}}=2,5$ and 10 . For these density ratios, the loop lengths $L_{\mathrm{c}}$ above which the global sausage mode becomes leaky are $6.66 \mathrm{Mm}, 13.33 \mathrm{Mm}$, and 19.99 Mm respectively (see the condition given by Eq. (8)). The corresponding values of the internal Alfvén speed were $C_{\mathrm{A} 0}=2.12 \mathrm{Mm} \mathrm{s}^{-1}$, $1.34 \mathrm{Mm} \mathrm{s}^{-1}$ and $0.95 \mathrm{Mm} \mathrm{s}^{-1}$, respectively. In all runs, the external Alfvén speed was kept the same, $C_{\mathrm{Ae}}=3 \mathrm{Mm} \mathrm{s}^{-1}$. This value corresponds to the available observational estimations (Nakariakov et al. 2003; Melnikov et al. 2005). For each density contrast ratio simulations were performed for loop lengths between $L=9 \mathrm{Mm}$ and $L=60 \mathrm{Mm}$. Thus, the wave length of the global sausage mode could be either shorter or longer than the cut-off wave length, allowing for the existence of trapped or leaky regime, respectively. The transverse size of the numerical domain was fixed at $150 \mathrm{Mm}$ and the numerical resolution was scaled with loop length to maintain a constant grid cell. 


\section{Results}

We performed a series of numerical experiments simulating the response of the slab to an initial perturbation of sausage symmetry in the transverse direction and having the global standing mode structure in the longitudinal direction as given by Eq. (13). The initial perturbation developed into a trapped or leaky global sausage mode oscillation, depending upon the loop length. We studied the dependence of the oscillation period and decay time upon the ratio of the slab length and width, and the density contrast ratio.

Figure 5 shows signals characteristic of trapped and leaky global sausage modes. The signal shown is the perturbation of the parallel $(z)$ component of the magnetic field at the loop apex. In the global sausage mode this is where this quantity has a maximum. The simulated loop has a density contrast ratio $\rho_{0} / \rho_{\mathrm{e}}=10$, for which the cut-off wave length is about $40 \mathrm{Mm}$. The upper panel is for a loop length of $15 \mathrm{Mm}$ (and, hence, the global mode wave length of $30 \mathrm{Mm}$ ), shorter than the cut-off length and thus supporting the trapped global sausage mode. The signal is therefore a harmonic oscillation with the frequency corresponding to the dispersion relation. The signal amplitude does not show any decay. There is also a long period modulation of this signal, due to the imperfect nature of the excitation and transverse boundary conditions. Such modulation is not seen if the mode is excited directly with the correct phase speed from the dispersion relation. For higher amplitudes, the beating could also be connected with resonant nonlinear coupling of the sausage mode to kink mode (see Nakariakov \& Oraevsky 1995, for discussion), but is negligible for the considered small amplitudes.

The lower panel of Fig. 5 is for a loop length of $60 \mathrm{Mm}$, corresponding to the global mode wave length of $120 \mathrm{Mm}$. This is far above the cut-off wave length of $40 \mathrm{Mm}$, so there is no trapped global mode, however a periodic signal is still clearly seen. The signal has two stages; an initial stage during which a fraction of the applied perturbation immediately leaks out of the loop (the signal "adjusts" itself to the leaky eigenmode), followed by a second stage in which the remaining perturbation is periodic and decaying because of the wave leakage. This second stage can be approximated as an exponentially damped harmonic oscillation.

Figures 6 and 7 show the dependence of the period of oscillation on the loop length for different density contrast ratios. For loop lengths which correspond to the global mode wave lengths shorter than the cut-off wave length, the period lies between the straight lines $2 L / C_{\mathrm{A} 0}$ and $2 L / C_{\mathrm{Ae}}$. For longer loops, the mode behaviour changes from the trapped one to the leaky one, and the period becomes less than but close to the value given by $2 L / C_{\mathrm{Ae}}$, and is in fact not dependent on the internal Alfvén speed at all.

Figure 6 also shows the dependence upon loop length of the signal quality. The quality is determined as the ratio of the decay time, estimated by fitting the oscillations to an exponential decay with decay constant $1 / \tau$, and the oscillation period. As expected, the signal quality increases with the density contrast ratio, i.e. denser loops are more efficient waveguides, but the rate of mode leakage is most strongly dependent on the loop length for the density contrast ratios considered. For each of the density contrast ratios oscillations continue to exist with detectable signal quality well above the theoretical cut-off.

Figure 8 shows the difference $(\Delta)$ between the period obtained by numerical simulation and that estimated from the loop length and the external Alfvén speed as

$P \simeq \alpha \frac{2 L}{C_{\mathrm{Ae}}}$

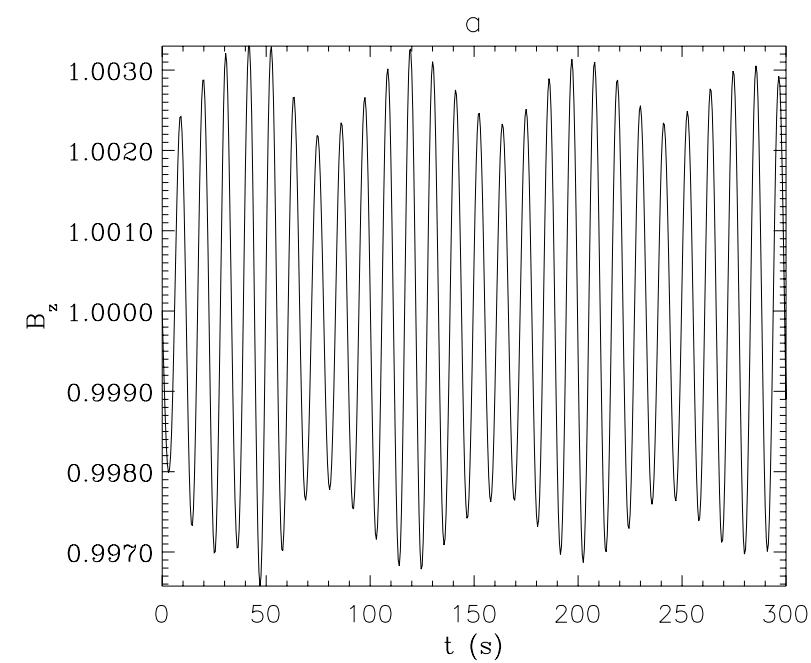

$\mathrm{b}$

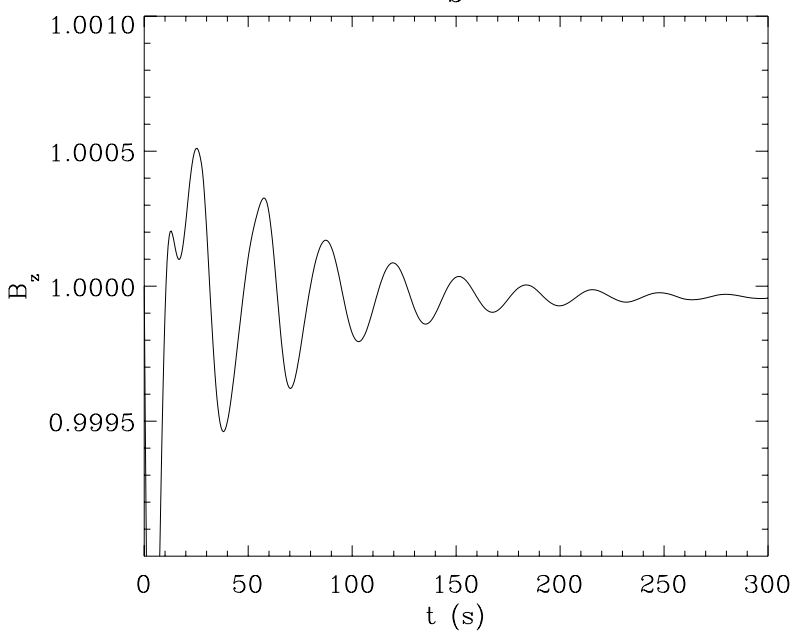

Fig. 5. Oscillations of the parallel component of the magnetic field at the loop apex for loops of length a) $15 \mathrm{Mm}$ and b) $60 \mathrm{Mm}$, corresponding to trapped and leaky modes respectively. The loop semi-width $a$ is $3 \mathrm{Mm}$ and the density contrast ratio is $\rho_{0} / \rho_{\mathrm{e}}=10$, for which the global sausage mode becomes leaky for lengths above $20 \mathrm{Mm}$.

where $\alpha<1$ and is chosen to be 0.95 in Fig. 8. Observations of this mode would provide us with seismological information about the Alfvén speed outside the loop, and hence the external magnetic field which cannot be measured directly.

\section{Conclusions}

Our direct full-MHD numerical modelling of global sausage oscillations of a low $\beta$ plasma slab demonstrated the following:

1. According to the slab model, the period of the global sausage mode depends upon the length of the loop in both trapped and leaky regimes, confirming the theory developed in Nakariakov et al. (2003).

2. Numerical modelling proves the existence of leaky global sausage modes in the case of long loops with small density contrasts.

3. The leaky global sausage modes are of detectable quality and thus can be responsible for quasi-periodic pulsations of flaring emission.

4. In the leaky regime, the period of the global sausage mode is almost independent of the internal Alfvén speed and is determined by the Alfvén speed outside the loop. 


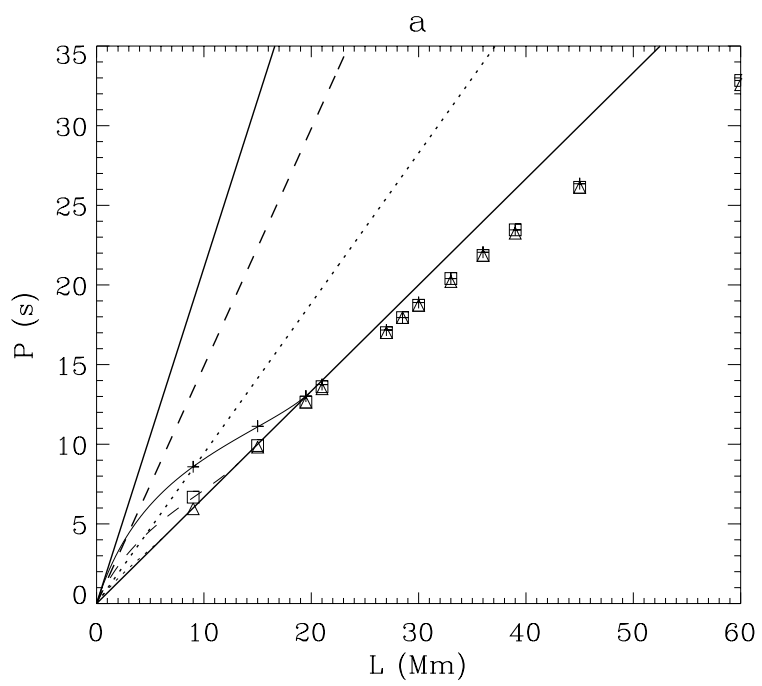

b

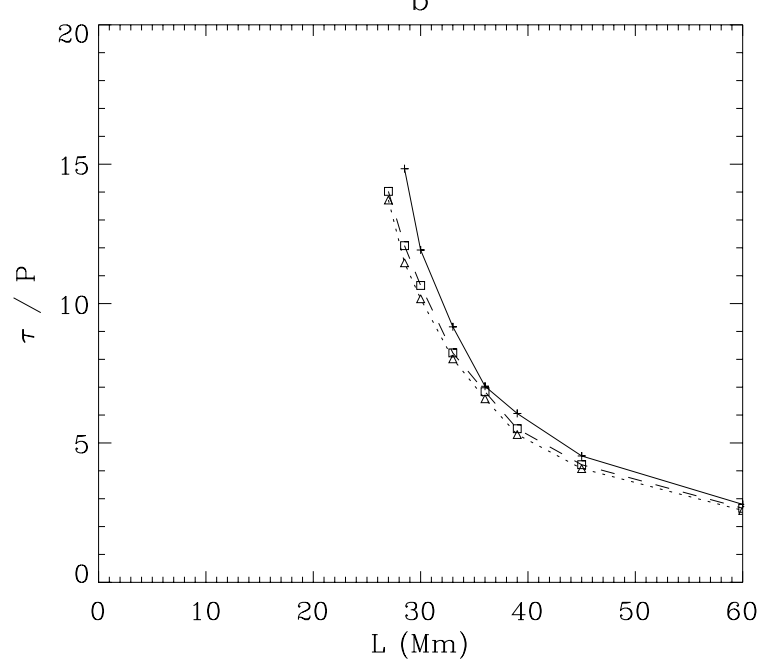

Fig. 6. a) The dependence of the period of oscillation upon the loop length for density contrast ratios of 2 (triangle), 5 (square) and 10 (cross). The dotted, dashed and solid curved lines correspond to the analytical dispersion relations for density contrast ratios of 2 , 5 and 10 respectively. The lower solid line corresponds to a period of $2 L / C_{\mathrm{Ae}}$. The dotted, dashed and upper solid lines correspond to the values of $2 L / C_{\mathrm{A} 0}$ for density contrast ratios of 2,5 and 10 respectively. b) The dependence of the signal quality upon the loop length. The quality is determined as the ratio of the decay time and the oscillation period.

5. The period of the global sausage mode provides us with seismological information about the Alfvén speed in the rarified inter-loop medium (outside the loop), and hence the external magnetic field which cannot be measured directly.

We conclude that the global sausage mode of a coronal loop can be responsible for pulsations with the periods in the range of 5-60 s, associated with solar and stellar flares (for typical loop lengths of 5-90 Mm and external Alfvén speeds of $\left.2-3 \mathrm{Mm} \mathrm{s}^{-1}\right)$. As the mode is essentially compressible, it can modulate thermal emission from the loop body (e.g. in soft $\mathrm{X}$-ray). Also, the density variations, as well as variations of the magnetic field produced by the mode, can modulate the radio emission. In loops which diverge with height, modulation of the longitudinal component of the magnetic field and hence of the mirror ratio, can produce periodic precipitation of non-thermal electrons, causing periodic modulation of the hard X-ray, white light and microwave emission coming from the dense plasma of

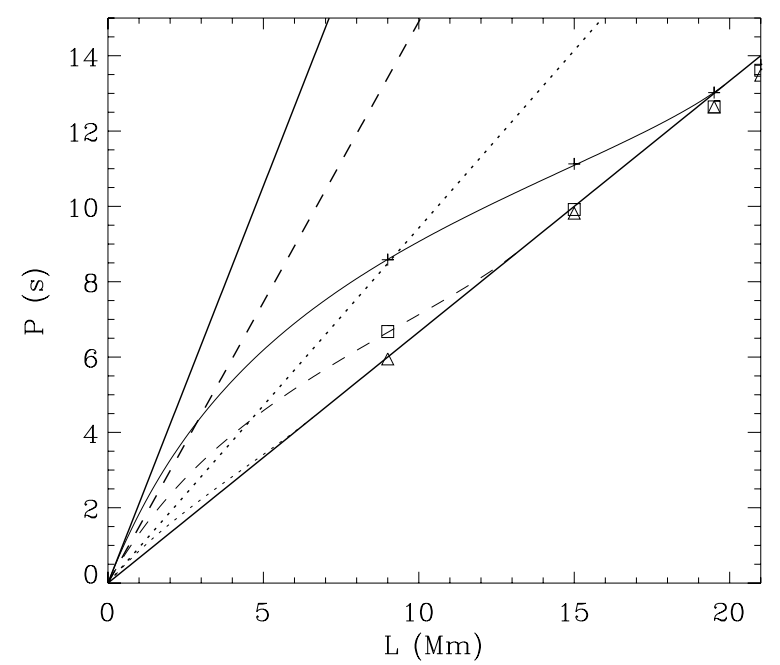

Fig. 7. A close-up of Fig. 6 for small loop lengths. In this limit the global sausage mode is trapped and period of oscillation is determined by the internal Alfvén speed.

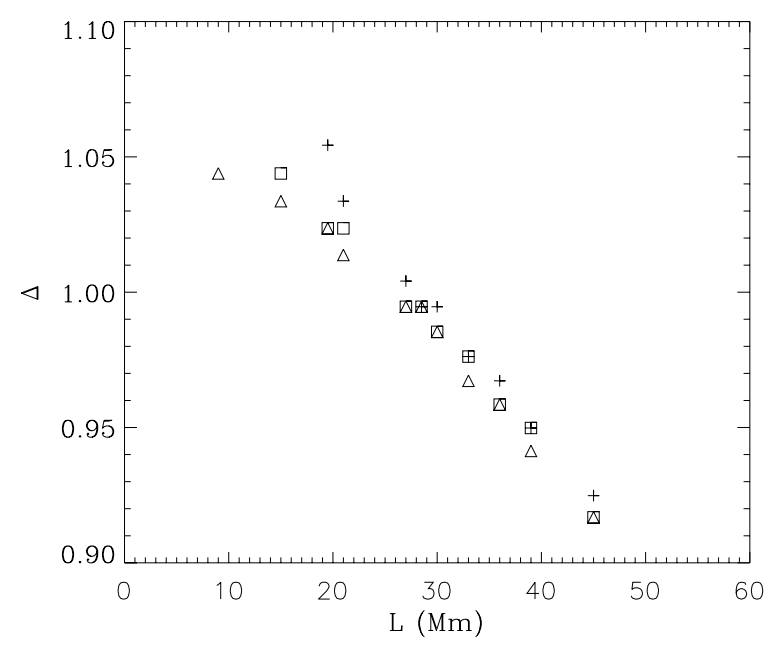

Fig. 8. The dependence upon the loop length of the difference $(\Delta)$ between the numerically obtained period and the period estimated using the external Alfvén speed. The results are for density contrast ratios of 2 (triangle), 5 (square) and 10 (cross).

the loop legs. Also, the mode can modulate the process of magnetic reconnection and electron acceleration connected with it, by the triggering mechanism proposed recently by Nakariakov et al. (2006).

Our results demonstrate that in both trapped and leaky regimes the period of standing sausage magnetoacoustic oscillations of coronal loops is determined by the length of the loop and hence is comparable with the longitudinal Alfvén transit time. Quasi-periodic pulsations with shorter periods, comparable with the transverse Alfvén transit time (e.g., with the periods of about a second, often reported in literature, see e.g. Stepanov et al. 2004) can possibly be explained in terms of higher spatial harmonics of this mode. However, it is not clear what is the mechanism responsible for the selection of those particular harmonics only. Simultaneous detection of several different higher harmonics could strengthen this interpretation. An alternative option for the generation of the shorter periods is the dispersively formed quasi-periodic fast wave train (Roberts et al. 1984; Nakariakov et al. 2004). 
In the trapped regime, the oscillation did not show any signs of decay, which is consistent with the theory developed by Nakariakov \& Roberts (1995) and Cooper et al. (2003). However, the discrepancy between the initial perturbation and the eigenmode leads to amplitude beating of the excited signal. The beating is not likely to be observed, because the signal would decay due to dissipative processes and other mechanisms which were neglected in the developed model.

Acknowledgements. D.J.P. was supported by a PPARC studentship.

\section{References}

Arber, T. D., Longbottom, A. W., Gerrard, C. L., \& Milne, A. M. 2001, JCP, 171, 151

Aschwanden, M. J. 2004, Physics of the Solar Corona (Berlin: Springer Praxis Books)

Aschwanden, M. J., Nakariakov, V. M., \& Melnikov, V. F. 2004, ApJ, 600, 458
Cooper, F. C., Nakariakov, V. M., \& Williams, D. R. 2003, A\&A, 409, 325 Edwin, P. M., \& Roberts, B. 1982, Sol. Phys., 76, 239

Kopylova, Y. G., Stepanov, A. V., \& Tsap, Y. T. 2002, Astron. Lett., 28, 783 Melnikov, V. F., Reznikova, V. E., Shibasaki, K., \& Nakariakov, V. M. 2005, A\&A, 439, 727

Nakariakov, V. M., \& Oraevsky, V. N. 1995, Sol. Phys., 160, 289

Nakariakov, V. M., \& Roberts, B. 1995, Sol. Phys., 159, 399

Nakariakov, V. M., \& Verwichte, E. 2005, Living Rev. Solar Phys., 2, 3, URL(05/07/2005): http: //www . livingreviews .org/lrsp-2005-3

Nakariakov, V. M., Melnikov, V. F., \& Reznikova, V. E. 2003, A\&A, 412, L7

Nakariakov, V. M., Arber, T. D., Ault, C. E., et al. 2004, MNRAS, 349, 705

Nakariakov, V. M., Foullon, C., Verwichte, E., \& Young, N. P. 2006, A\&A, 452, 343

Ofman, L. 2005, Space Sci. Rev., 120, 67

Roberts, B., Edwin, P. M., \& Benz, A. O. 1984, ApJ, 279, 857

Rosenberg, H. 1970, A\&A, 9, 159

Stepanov, A. V., Kopylova, Y. G., Tsap, Y. T., et al. 2004, Astron. Let., 30, 480

Zaitsev, V. V., \& Stepanov, A. V. 1975, Issled. Geomagn. Aeron. Fiz. Solntsa, 37,3

Zaitsev, V. V., \& Stepanov, A. V. 1982, Sov. Astron. Lett., 8, 132 